\title{
SEQUENCE-BASED AND MONOCLONAL ANTIBODY TYPING OF LEGIONELLA PNEUMOPHILA ISOLATED FROM PATIENTS IN PORTUGAL DURING 1987-2008
}

\author{
M J Chasqueira (mjchasqueira@fcm.unl.pt) ${ }^{1}$, L Rodrigues ${ }^{1}$, M Nascimento $^{2}$, T Marques ${ }^{1,2,3}$ \\ 1. Microbiology Department, Chronic Diseases Research Centre - CEDOC, Faculty of Medical Sciences, “FCM-UNL”, Lisbon, Portugal \\ 2. Microbiology Laboratory, Santa Cruz Hospital "HSC-CHLO", Carnaxide, Portugal \\ 3. Coordinator of the Legionnaires' Disease Integrated Epidemiological Surveillance Programme in Portugal
}

\begin{abstract}
The monoclonal antibodies and the sequence-based typing (SBT) are two methodologies widely used to characterise Legionella pneumophila strains serogroup 1 (sg1). In this study, we analysed the clinical strains received in two Portuguese laboratories since 1987, including the strains isolated in Portugal during the four years of the surveillance scheme for Legionnaires' disease implemented in 2004. In total, 63 clinical isolates of L. pneumophila sg1 were differentiated by SBT into 19 different sequence types. Ten of them were new in the SBT database of the European Working Group for Legionella Infections (EWGLI). As a result of the combination of the two methodologies, these strains were discriminated into 25 different profiles. This study enabled, for the first time in Portugal, not only to characterise the L. pneumophila sg1 clinical isolates, but also to create a database of Portuguese profiles for use in epidemiological surveillance efforts.
\end{abstract}

\section{Introduction}

Legionella pneumophila is a Gram-negative facultative intracellular pathogen, which is responsible for Legionnaires' Disease. This microorganism has increasingly been recognised as an important cause of pneumonia since its first description in 1977 [1]. The characterisation of clinical isolates of L. pneumophila enables us to learn about its epidemiology in a certain geographic region, as well as to create a database of circulating profiles [2-7].

The combination of a genotypic method with monoclonal antibody (MAb) typing has been described as a useful approach for epidemiological typing of L. pneumophila isolates [7-10]. MAbs of the Dresden panel allow subdividing the serogroup 1 of L. pneumophila as having, or not having, the epitope recognised by the MAb 3/1. According to epidemiological studies, this epitope appears to be associated with virulence [11]. Sequence-based typing (SBT) is one of the genotypic methods that can be applied for this purpose. It was adopted as an international standard and is widely used by the members of the European Working Group for Legionella Infections (EWGLI), since it is a simple, rapid and discriminatory typing method. Furthermore, it also allows the exchange of data between laboratories $[7,8]$.

In 1999, the Portuguese public health authorities implemented a surveillance scheme for Legionnaires' Disease based on clinical reports. Later, in 2004, a surveillance scheme based on laboratory notifications was added. The Legionella laboratory in the microbiology department of the Faculty of Medical Sciences in Lisbon and the National Institute of Health Dr Ricardo Jorge (INSA) are the two laboratories involved in this surveillance scheme.

The aim of this study was to investigate the distribution of sequence types (ST) and monoclonal antibody subtypes among clinical isolates of L. pneumophila in Portugal.

\section{Material and methods}

As far as the present study is concerned, the SBT methodology, using seven genes (flaA, pilE, asd, mip, mompS, proA and neuA), was applied to 63 clinical isolates of L. pneumophila serogroup 1 (sg1), and four from non-sg1 (one isolate was sg 10, another was sg 12 and the two remaining reacted with "Legionella pneumophila serogroups 2-14 Latex Test Reagent" (Oxoid), but the serogroup could not be determined using our MAbs protocol) (see Table). The L. pneumophila strains were typed with MAbs of the Dresden panel, by using an indirect immunofluorescence test $[10,11]$.

We analysed the clinical strains received since 1987 by the laboratories of Santa Cruz Hospital and the Faculty of Medical Sciences, including the 19 strains isolated during the four years of the surveillance scheme for Legionnaires' disease. In total, 67 strains were sent for typing by 17 Portuguese hospitals. Thirty of them were isolated from patients with nosocomial infections and 20 from patients with community-acquired infections; the remaining 17 had an undetermined origin.

The genomic DNA used for the SBT method was extracted with the InstaGene Matrix kit (Bio-Rad), and the PCR amplification was performed by using puRe Taq Ready-to-Go beads (Amersham Biosciences). The primers and the PCR conditions were the same as those used by Gaia et al. and Ratzow et al. [7,8,12]. After purification with the Qiaquick PCR purification Kit (Qiagen), both strands of the amplicons were sequenced by StabVida on a 3700 ABI DNA sequencer (Applied Biosystems) using the Big Dye terminator DNA sequencing kit. The nucleotide sequences obtained were compared to those in EWGLI-SBT database [13]. All putative new sequences were confirmed before being sent to the curators of the database. 


\section{Results}

In this study, all but three of the strains included were typable by SBT using the seven genes (see Table). The neuA primers failed to type these three strains, all of which were non-sg1 (one sg 10 and the other two could not be identified with MAbs of Dresden panel),suggesting that neuA primers described by Ratzow et al. [12] are not always suitable for serogroups other than sg1. Other teams have also reported amplification problems with the neuA primers [14].

Applying SBT, the sample was discriminated into 7, 7, 11 , $8,12,7$ and 7 types, based on the sequences of $\mathrm{flaA}$, pilE, asd, mip, mompS, proA and neuA, respectively. As a consequence, the 67 isolates were divided into 23 STs in total. The distribution was as follows: the 63 L. pneumophila sg1 isolates were included into $19 \mathrm{ST}$, and the four L. pneumophila non-sg1 isolates into the remaining four ST.

Ten of the 19 STs from L. pneumophila sg1 and the four STs from L. pneumophila non-sg1 were different from the ones that already existed in the EWGLI-SBT database. In addition, six new allele numbers (22 and 29 for the mip gene, and 24, 20, 34 and 23 for the pilE, asd, mompS and proA genes, respectively) were assigned by the curators after our data were submitted to the database. It is interesting to notice that five of these new allele numbers were detected only in L. pneumophila non-sg1 strains

T A B L E

Twenty-three SBT profiles of 67 Portuguese L. pneumophila clinical isolates, 1987-2008

\begin{tabular}{|c|c|c|c|c|c|c|}
\hline \multirow[b]{2}{*}{ ST } & \multirow[b]{2}{*}{ Allelic profile ${ }^{a}$} & \multirow[b]{2}{*}{ No. of strains } & \multicolumn{3}{|c|}{ Dresden panel } & \multirow[b]{2}{*}{ Epidemiological relatedness } \\
\hline & & & Serogroup & MAb subgroup & $\begin{array}{l}\text { No. of } \\
\text { strains }\end{array}$ & \\
\hline \multirow{2}{*}{$100^{b}$} & \multirow{2}{*}{$3,8,1,10,14,12,2$} & \multirow{2}{*}{32} & \multirow{2}{*}{1} & Allentown/France & 18 & \multirow{2}{*}{ Related } \\
\hline & & & & Philadelphia & 14 & \\
\hline \multirow{2}{*}{1} & \multirow{2}{*}{$1,4,3,1,1,1,1$} & \multirow{2}{*}{3} & \multirow{2}{*}{1} & Philadelphia & 2 & \multirow{2}{*}{ Unrelated } \\
\hline & & & & Olda & 1 & \\
\hline \multirow{2}{*}{23} & \multirow{2}{*}{$2,3,9,10,2,1,6$} & \multirow{2}{*}{3} & \multirow{2}{*}{1} & Philadelphia & 2 & \multirow{2}{*}{ Unrelated } \\
\hline & & & & Knoxville & 1 & \\
\hline \multirow{2}{*}{62} & \multirow{2}{*}{$8,10,3,15,18,1,6$} & \multirow{2}{*}{3} & \multirow{2}{*}{1} & Allentown/France & 2 & \multirow{2}{*}{ Unrelated } \\
\hline & & & & Philadelphia & 1 & \\
\hline $103^{b}$ & $1,4,3,22^{\mathrm{b}}, 1,1,1$ & 3 & 1 & Philadelphia & 3 & Unrelated \\
\hline 20 & $2,3,18,15,2,1,6$ & 2 & 1 & Knoxville & 2 & Unrelated \\
\hline \multirow{2}{*}{42} & \multirow{2}{*}{$4,7,11,3,11,12,9$} & \multirow{2}{*}{2} & \multirow{2}{*}{1} & Knoxville & 1 & \multirow{2}{*}{ Unrelated } \\
\hline & & & & Benidorm & 1 & \\
\hline 14 & $4811101012=$ & 2 & 1 & Allentown/France & 1 & Unnolatod \\
\hline 44 & $4,8, \perp \perp, \perp U, \perp U, \perp<,\llcorner$ & 2 & 1 & Philadelphia & 1 & Unretated \\
\hline $99^{b}$ & $4,8,11,5,29,12,10$ & 2 & 1 & Knoxville & 2 & Unrelated \\
\hline & & & & Philadelphia & 1 & \\
\hline $101^{\circ}$ & $6,10,15,15,21,4,6$ & 2 & 1 & Knoxville & 1 & Unrelated \\
\hline 16 & $2,10,18,10,2,1,9$ & 1 & 1 & Knoxville & 1 & \\
\hline 22 & $2,3,6,10,2,1,6$ & 1 & 1 & Philadelphia & 1 & \\
\hline 94 & $12,8,11,5,20,12,2$ & 1 & 1 & Knoxville & 1 & \\
\hline $98^{b}$ & $8,10,3,10,2,5,6$ & 1 & 1 & Philadelphia & 1 & \\
\hline $102^{b}$ & $8,19,5,15,18,5,10$ & 1 & 1 & Philadelphia & 1 & \\
\hline 146 & $2,10,18,10,2,1,6$ & 1 & 1 & Philadelphia & 1 & \\
\hline $172^{b}$ & $1,4,3,1,1,1,2$ & 1 & 1 & Philadelphia & 1 & \\
\hline $173^{\mathrm{b}}$ & $6,10,14,15,21,4,6$ & 1 & 1 & Knoxville & 1 & \\
\hline $174^{b}$ & $4,8,11,5,10,12,15$ & 1 & 1 & Allentown/France & 1 & \\
\hline $153^{b}$ & $2,10,3,28,9,14,3$ & 1 & 12 & --- & & \\
\hline $\mathrm{c}, \mathrm{b}$ & $6,10,21,28,4,14,0$ & 1 & 10 & --- & & \\
\hline $\mathrm{c}, \mathrm{b}$ & $2,24,20,29,34,23,0$ & 1 & d & --- & & \\
\hline $\mathrm{c}, \mathrm{b}$ & $3,24,1,29,34,23,0$ & 1 & d & --- & & \\
\hline
\end{tabular}

SBT: sequence-based typing; ST: sequence type.

a Sequence of genes flaA, pile, asd, mip, momps, proA, neuA.

b New profiles and allele numbers are in bold.

c Problems in amplifying the neuA gene.

d Strain reactive with "Legionella pneumophila serogroups 2-14 Latex Test Reagent" (Oxoid). Serogroup could not be determined using MAbs of Dresden panel. 
that were non-typable with MAbs from the Dresden panel (see Table). The ST100 $(3,8,1,10,14,12,2)$ was the most frequent allele (32/67). This is a new profile and all of the ST100 strains had been isolated in patients of the same hospital over a period of several years. Twenty-four of the 32 strains with this profile came from nosocomial infections and the remaining eight from undetermined origin. These eight patients had subjacent diseases and needed hospital care frequently, suggesting that some or even all of these sporadic cases could be hospital-acquired, too. The STs 1, 20, 23, 42, 44, 62, 99, 101 and 103 were found in more than one strain. The 22 strains belonging to these nine STs were unrelated according to their source origin. In this study, ST1 $(1,4,3,1,1,1,1)$, the most frequent profile reported in the world, was found only in three isolates (see Table).

The 19 strains sent by the surveillance scheme during the past four years, showed high profile diversity. Eleven distinct STs were obtained, five of them for the first time in Portugal. These strains were isolated in 11 different hospitals, five, four and two, respectively, from the north, the centre and the south of Portugal. The majority of the isolates came from community-acquired infections (12/19).

Using the Dresden panel of MAbs, the 63 L. pneumophila sg1 strains had previously been divided into five different subgroups (unpublished data). All strains but one possessed the virulence-associated epitope recognised by MAb 3/1 [11], and the Philadelphia subgroup was the most frequent with 28 of the 63 strains (see Table). As a result of the combination of the two methodologies, MAbs and SBT, these strains were now differentiated into 26 different profiles. The results showed that the Philadelphia subgroup was the most heterogeneous as it was divided into 12 different STs. On the other hand, identical STs were found among strains reactive with different MAbs (see Table). These two facts support the idea that it is valuable to add genotyping methods to MAb typing when defining profiles within a phenotypic subgroup $[7,9]$.

\section{Discussion}

As far as our experience is concerned, the SBT scheme is technically simple for a laboratory with basic molecular expertise and equipment, provided that there is access to a sequencing laboratory. Although this method proved to be a good genotypic method for epidemiological investigations, showing unambiguous results that are easy to interpret [4,6-8], one of the limitations of the epidemiological studies is the fact that most diagnoses are made by urinary antigen test, without strain isolation. The EWGLI 2008 database showed that culture was the methodology used in only 62 of the 866 reported cases in the 35 countries participating in EWGLINET [15]. In Portugal, the data were similar: in the past four years, the strain was isolated for only 19 of 237 Legionella notifications (unpublished data). Thus, Legionella isolates are not available for the majority of cases and therefore the results of this study may not entirely reflect the distribution of the Legionella strains responsible for the disease in Portugal. However, our collection contains the majority of the clinical isolates collected in Portugal since 1987; so it is possible that this sampling is representative of the profiles circulating in Portugal.

The significant profile diversity we observed is in accordance with reports from the other countries $[4,14,16,17]$. Due to the relatively low number of isolates in each ST, with the majority
$(13 / 23)$ of the STs being detected only once, it is not possible to establish a correlation between the ST and the infection origin.

To summarise, this study enabled us, for the first time in Portugal, to characterise the L. pneumophila clinical isolates with SBT methodology and MAbs, as well as to create a database of Portuguese L. pneumophila profiles for use in epidemiological surveillance efforts. It was also a contribution to the EWGLI-SBT database and to the knowledge of the European L. pneumophila diversity, owing to the high rate of new STs obtained.

\section{Acknowledgements}

We thank colleagues of the Microbiology Laboratory INSA for sending some of the strains isolated in the Legionaries' Disease Integrated Epidemiological Surveillance Programme.

We also thank Ana Margarida Farinha for revision of the manuscript.

\section{References}

1. Stout JE, Rihs JD, Yu VL. Legionella. In: Murray PR Baron EJ, Jorgensen JH, Pfaller MA, Yolken RH, editors. Manual of clinical microbiology. 8th ed. Washington, DC: American Society for Microbiology, 2003. p. 809-23.

2. Gilbert G. Molecular diagnostics in infectious diseases and public health microbiology: cottage industry to postgenomics. Trends Mol Med. 2002; 8(6):280-7.

3. Marques MT. Contribuição para o estudo do género Legionella e sua ocorrência em Portugal. [Contribution for the better knowledge of the genus Legionella and its occurrence in Portugal]. PhD Thesis. Arquivos do Instituto Bacteriológico Câmara Pestana. [Archives of the Bacteriological Institute of Câmara Pestana]. ISSN 0365-2998. 1999;XXIII:89-216. [In Portuguese].

4. Amemura-Maekawa J, Kura F, Chang B, Watanabe H. Legionella pneumophila serogroup 1 isolates from cooling towers in Japan form a distinct genetic cluster. Microbiol Immunol. 2005; 49(12):1027-33.

5. Urwin R, Maiden MC. Multi-locus sequence typing: a tool for global epidemiology. Trends Microbiol. 2003;11(10):479-87.

6. Scaturro M, Losardo M, de Ponte G, Ricci M. Comparison of three molecular methods used for subtyping of Legionella pneumophila strains isolated during an epidemic of legionellosis in Rome. J Clin Microbiol. 2005;43(10):5348-50.

7. Gaia V, Fry N, Harrison T, Peduzzi R. Sequence-Based Typing of Legionella pneumophila serogroup 1 offers the potential for true portability in Legionellosis outbreak investigation. J Clin Microbiol. 2003; 41(7):2932-9.

8. Gaia V, Fry N, Afshar B, Luck C, Meugnier H, Etienne J, et al. Consensus sequencebased scheme for epidemiological typing of clinical and environmental isolates of Legionella pneumophila. J Clin Microbiol. 2005;43(5):2047-52.

9. Bernander S, Jacobson K, Helbig J, Luck P, Lundholm M. A hospital-associated outbreak of Legionnaires' disease caused by Legionella pneumophila serogroup 1 is characterized by stable genetic fingerprinting but variable monoclonal antibody patterns. J Clin Microbiol. 2003;41(6):2503-8.

10. Helbig J, Kurtz J, Castellani Pastoris M, Pelaz C, Luck P. Antigenic lipopolysaccharide components of Legionella pneumophila recognized by monoclonal antibodies: possibilities and limitations for division of the species into serogroups. J Clin Microbiol. 1997;35(11):2841-5.

11. Helbig J, Bernander S, Castellani Pastoris M, Etienne J, GaiaV, et al. PanEuropean study on culture-proven Legionnaires'disese: distribution of Legionella pneumophila serogroups and monoclonal subgroups. Eur J Clin Microbiol Infect Dis. 2002;21(10):710-6.

12. Ratzow S, Gaia V, Helbig JH, Fry NK, Lück PC. Addition of neuA, the gene encoding $\mathrm{N}$-acylneuraminate cytidylyl transferase, increases the discriminatory ability of the consensus sequence-based scheme for typing Legionella pneumophila serogroup 1 strains. J Clin Microbiol. 2007;45(6):1965-8.

13. European Working Group for Legionella Infections. The EWGLI SBT database for the typing of Legionella pneumophila. [Accessed June 2008]. Available from: http://www.hpa-bioinformatics.org.uk/legionella/legionella_sbt/php/ sbt_homepage.php

14. Harrison TG, Afshar B, Doshi N, Fry NK, Lee JV. Distribution of Legionella pneumophila serogroups, monoclonal antibody subgroups and DNA sequence types in recent clinical and environmental isolates from England and Wales (2000-2008). Eur J Clin Microbiol Infect Dis. 2009; 28(7):781-91. Epub 2009 Jan 
15. European Working Group for Legionella Infections. Cases by Year of Onset and Main Method of Diagnosis. [Accessed December 2008]. Available from: http:// www.ewgli.org/data/data_tables/year_onset_method_diagnosis.asp

16. Borchardt J, Helbig JH, Lück PC. Occurrence and distribution of sequence types among Legionella pneumophila strains isolated from patients in Germany: common features and differences to other regions of the world. Eur $\mathrm{J}$ Clin Microbiol Infect Dis. 2008; 27(1):29-36.

17. Kozak NA, Benson RF, Brown E, Alexander NT, Taylor TH Jr, Shelton BG, et al. Distribution of lag-1 alleles and sequence-based types among Legionella pneumophila serogroup 1 clinical and environmental isolates in the United States. J Clin Microbiol. 2009; Jun 24. [Epub ahead of print].

This article was published on 16 July 2009.

Citation style for this article: Chasqueira MJ, Rodrigues L, Nascimento M, Marques T. Sequence-based and monoclonal antibody typing of Legionella pneumophila isolated from patients in Portugal during 1987-2008. Euro Surveill. Available online: http://www.eurosurveillance.org/ViewArticle.aspx?ArticleId=19271 